\title{
EDITORIAL
}

\section{Quality Control in Pathology}

For many years, forms of Quality Control (QC) have been an integral part of the Practice of Laboratory Medicine, and for many years, Army laboratories the world over have participated fully in such QC schemes.

Perhaps it is easiest to see how the system works in the principally numerical reporting of Clinical Chemistry. Sera are distributed by the controlling laboratory to the participants. The constituents in question are assayed locally and the results are returned to the distributor who then analyses them statistically. This analysis tells the participating laboratory how well it is doing with regard to accuracy and precision, and overall in comparison to other participating laboratories. Such information may be of great help to laboratory managers; for example, small changes in the precision of a particular parameter should lead to local investigation and identification of problems before these become clinically relevant.

Similar QC programmes exist in other disciplines such as Haematology and Medical Microbiology. In the former, because of the proportion of numerical results, the programme is in part similar to that in Clinical Chemistry. In Haematology, however, if all aspects of practice are to be controlled, blood films must also be distributed as part of the programme; the introduction of diagnostic opinion into the system presupposes diagnostic consensus by the controlling laboratory, and in practice there has been little problem in achieving this. A further problem arises in the control of Medical Microbiology in that much of the material distributed must include living organisms which are subject to biological changes, including the death of the organisms. The distributing laboratory overcomes artefacts arising from this by sending samples of the QC material to itself by the same means as was used to send it to the participating laboratories and retesting this material. Again, in practice, neither the problems of distribution of living organisms nor of assessing results in this discipline have proved insurmountable.

Such systems of QC are not foolproof and are always open to misuse. It is virtually impossible to introduce the $\mathrm{QC}$ material into the laboratory without its being recognised by the technician on the bench; it is thus liable to be treated differently from the other specimens. It may be subjected to multiple testing to achieve better accuracy and precision. It may be tested by a more senior technician than the one who is responsible for handling the clinical material. Groups of laboratories may contact one another and pool information about QC results supposedly to the statistical benefit of the group. Such abuses, however, are easily prevented by good laboratory management, and are certainly not widespread in Army Pathology.
One of the factors which has contributed to the almos universal acceptance of QC has been anonymity; particio pating laboratories are, of course known to the organi sers of the programmes, but are identified in cumulativ $\overrightarrow{\bar{p}}$ reports only by coded numbers, and cannot therefore bo identified by other participants. Only in the uncommo event of persistently poor performance is an individuac. laboratory identified, and then, only to those responsi $\bar{\Phi}^{\circ}$ ble for the work of that laboratory; in the Army sucla identification would be to the Director of Army Pathology.

In summary then, adequate QC schemes exist fo $\overrightarrow{\vec{\omega}}$ several disciplines in laboratory medicine and these are widely used and of great benefit to those working in anç using pathology laboratories.

In this issue of The Journal, Whitehead et al address the difficult question of diagnostic QC in Histopathow logy. There is currently no national system of $Q C$ in this discipline, nor indeed any consensus as to how such in scheme could operate. A DHSS Committee is currently, considering the problem, and correspondence on tho subject is to be found regularly in the Bulletin of Royal College of Pathologists.

The completed histopathology report is the prod范苑 of numerous procedures. The process begins with कृष्ठ surgical operation, and factors such as fixation, sampl k़్ in the laboratory, processing, cutting on the microtore and staining and mounting must all be correcto performed if the slides to be examined are to lead to correct diagnosis. Even when these essentially technica procedures are carried out in an exemplary manner other factors such as the given clinical history and the experience of the reporting pathologist are crucia $\vec{B}$ variables. Although some of these procedures can be automated - processing and staining, for example - the majority depends on the individual skill of the technician? and the pathologist applied individually to each specimen.

Now, when assessing the performance of a Clinica Chemistry laboratory where much of the work i.t. undertaken by automated apparatus, it is valid to sup pose that good performance on one specimen in a large batch reflects good performance on all the specimens in that batch, and probably good performance on all thein specimens tested by that method. From the foregoing however, it will be clear that such assumptions are not valid for Histopathology; good performance on materia? circulated as part of a QC programme, and inevitablos recognised as such by both technical and medical staff may indicate very little about overall performance foro other material.

There are also practical problems associated with any national QC programme in histopathology which ${ }^{\omega}$ attempts to operate by distributing material and 
analysing returns. What material is to be distributed? If stained and mounted slides are distributed, all of the important stages of preparation before the presentation of slides to the pathologist are out with the QC scheme, but it is not possible to distribute tissue as the quantity of tissue required would almost certainly be greater than its availability in any given case. If more than one case were used, direct comparison, and the establishment of a numerical measure of adequate performance, would be impossible.

Then there is the problem of specialisation within histopathology. All practising histopathologists use from time to time the services of colleagues with special skills and experience in particular fields. Material from particular problematic cases is referred for further opinion at the discretion of the local pathologist, and this system works well and to the benefit of the patients in question. Might it be that in the situation of a national QC programme that the histopathologist working primarily in gynaecological oncology would feel obliged to express an opinion on dermatological pathology contrary to his normal practise? Such opinions would hardly reflect the quality of his day-to-day work, but a reply that the case would be referred to a colleague would also be difficult to evaluate in practise.

Finally, there is the problem of maintaining anonymity, an important factor in the success of QC programmes in other disciplines. By definition, histopathology reports are signed by their authors. It is easy to say that this rule could be waived in the case of QC reports, and a code number substituted, but in practice, it is not at all difficult to find out which pathologist issued a given report. The possibility of individual adverse comment may well lead to the nonparticipation of those pathologists who most need the benefits of diagnostic QC.

In their article, Whitehead and his colleagues outline the functions of the Army Histopathology Registry (AHR) and elaborate on one aspect of these - diagnostic quality control. The AHR has existed now for some forty years, and its staff have been responsible for reexamining histopathological material from all military hospitals over that time; there is therefore considerable experience in this field, and over this time there has been very little resentment of the sytem by contributing pathologists. This has been due in no small part to the sensitivity of the AHR staff, and their emphasis on diagnostic assistance and educative comment on their reports to contributors. In short, the system works well for the patients and clinicians, and for many years has practised effective diagnostic QC in histopathology.

Might it be that here is a model which could be adapted for use by the much larger civilian community? It is certainly not possible to envisage some National Histopathology Registry reviewing all the work of every histopathologist in practice in Great Britain, but it is not too difficult to envisage locally organised Registries reviewing a proportion of all cases reported by participating laboratories. The cases could be referred at random by simply requesting slides and a report form, for example, every fifticth case selected by serial laboratory number. Such a system would ensure that both the mundane and the esoteric were reviewed, and would therefore relate fairly closely to the normal work of the contributing laboratory. The quality of the technical work could be assessed by suitable laboratory technicians, and the diagnostic review would be conducted by pathologists within the group. Responsibility for reviewing this material could rotate within the group so that no individuals nor organisations carried the burden for too long. If suitable sensitivity and attention to helpfulness were shown by the reviewers, the absence of anonymity should not pose a problem. Similar groups could be organised specifically for sub-specialties on a larger geographical basis. National co-ordination would, of course, be necessary.

Whatever system is eventually adopted for QC in histopathology by our civilian colleagues, those using the services of histopathologists in the Army Medical Services should be reassured that effective QC in this discipline is currently exercised to the benefit of our patients, clinicians and histopathologists. Indeed, if it were felt that the concept of QC had a wider application to other disciplines of medicine, particularly those demanding subjective judgement, the system for histopathology outlined in this editorial might well provide a model. 